\title{
Rationale for a New Journal: the Astronomy Theory, Observations and Methods (ATOM) Journal
}

\author{
Michael Fitzgerald ${ }^{1 *}$
}

\begin{abstract}
In this article, the rationale behind the creation of a new journal, Astronomy Theory, Observations and Methods (ATOM) journal, currently hosted at rtsre.org is provided. It aims to fill a niche in the community for papers on any general topic in astronomy that may not find their place in top tier astronomical journals. The article outlines the thinking behind why there is a gap to be filled with regards to current scholarly metrics and the nature of other journals of similar scope and impact. The journal aims to be accessible to new and novice scientific authors, as well as those more established, through accessible developmental peer review and an explicit aim to avoid using publication metrics as a barrier to publication selection. The scope, which accepts more broader articles than most, of the journal and considerations on behalf of a potential author are also outlined.

\section{Keywords}

Scientific publishing - Amateur Astronomy - Student Research - Scientometrics

${ }^{1}$ School of Education, Edith Cowan University

${ }^{*}$ Corresponding author: psyfitz@gmail.com
\end{abstract}

\section{Introduction}

Part of the rationale for many astronomy student research projects (e.g. Fitzgerald et al. 2014; Percy 2018; Swift and Vyhnal 2018; Cutts 2018; Gomez and Fitzgerald 2017; Freed 2018) is that the students will be undertaking real research with real data with real scientists. If these projects are particularly 'real', then this activity should, by natural extension, be able to be published in a 'real' journal. It is also true, that a similar line of argument exists in the pro-amateur (pro-am) community, where the backyard astronomer is seen as someone who is readily capable of contributing important observations to science (Buchheim 2007; Conti 2018). However, it is generally not the case that much of this research from either of these two communities ends up in a mainstream professional astronomy journal, such as MNRAS, ApJ, AJ or AA, PASP or PASA.

The "race to the top" for mainstream astronomy journals means that the journals in the middle to top range of impact factor, such as those mentioned above, tend to reject articles that only have minimal, low or moderate impact. This is normal and, in some places, explicitly stated (e.g. Bertout and Schneider 2004). While it is relatively rare, it is entirely possible that if the research is taken to its natural extent in an area of sufficient interest to the astronomical community then such authentic projects undertaken by students, teachers and pro-ams can be published in middle to top tier astronomical journals. Examples include (Beuermann et al. 2009, 2011; Backhaus et al. 2012; Frew et al. 2011; Fitzgerald et al. 2012, 2015; Guieu et al. 2010; Howell et al. 2006, 2008; Rebull et al. 2011).

It is, however, nearly a truism that most research stemming from pro-am and education endeavours 
is typically not going to reach such a level. This research would be usually one of the first research experiences the authors may have and hence they likely will not have the depth of experience, skills and knowledge that someone in their post-PhD career might have. Therefore, the research will likely be smaller in scope and less on the cutting edge of science than articles that may end up in a higher end journal. Much of the time, the research may not be accepted in low to medium impact journals either.

In this paper, the rationale is outlined for a new astronomy research journal accepting of all impact levels of articles without an explicit goal of maximising impact factor or citation rates. We first provide an overview of the current state of mainstream astronomical journals and the quantitative ratings that drive them, their authors and the institutional policy that drives the activity of authors. We next present other journals that are at a similar level as the intended new journal that are already accepting such articles. We then provide an overview of the new journal, its scope, requirements and peer review process. We finish up with important considerations that an author should make when deciding to publish in this journal.

\section{Current metrics of astronomy authors and journals}

In order to, at least partially, understand why there is a gap to be filled in the suite of astronomy journals available to potential authors, it is necessary to understand the underlying metrics driving publication decisions by both journals and researchers. Here we focus on the primary individual researcher metric, the h-index, and the primary journal metric, the impact factor.

\section{The $\mathrm{h}$-index}

The manner in which a researcher is rated and ranked against their peers has been changing over time. In 2004, the h-index did not yet exist. Researchers were generally rated by the total number of publications and the total number of citations. This slowly started changing when Hirsch (2005) presented his idea of quantifying a researcher's scientific output in a single index, $h$. The simplest explanation is that a researcher has a h-index of value $h$, where $h$ is the nth paper sorted by citation that has $\mathrm{n}$ citations. In 2018, this index has become ubiquitous where various versions of the $\mathrm{h}$-index are presented as an indicative proxy for a researcher's worth. This will likely change in the future as more network modelling and artificial intelligence driven metrics appear but, for the moment, it is currently the central scholarly metric for individual researchers.

\section{The impact factor}

Similarly, journals have their own central metric, the "impact factor", which is typically taken as the number of citations over the last two years divided by the number of articles published in the same period of time.

Impact Factor $=$ Total Citations in last two years / Publications in last two years

This value is used to create leaderboards or ranking tables of journals. One of the most prominent journal ranking systems being Scopus. In turn these leaderboards and rankings are used in a variety of ways by institutions and governments around the world to rate the research capacity of institutions (and hence distribute research funding). To complete the loop, these institutions then put pressure on their researchers to only publish in journals that exist towards the top of the leaderboard. This means that for journals to attract the best papers and to score highly on the leaderboard, they need to reject as many low-moderate level papers as possible.

Some journal articles, however, may never be intended to get more than a couple of citations. The results from these papers may be intended to be amalgamated into larger databases and review papers, leading quickly to the situation where their citation links are lost or undercited, especially for updates of single objects or papers with null results. The article may be read broadly by the community and inform much scholarly activity and conversation but not be of a nature that it gathers many 
citations. It may also be the case that a paper takes many years to be cited significantly and hence does not contribute to the journal impact factor in the two year period where this is calculated. This does not make these papers not valuable. It is also the case that just because a paper gets a citation, that is not necessarily an indicator of quality - the citing paper can easily be saying the cited paper was incorrect.

\section{All that is metric is not gold?}

These two metrics, the h-index and the impact factor, do not necessarily describe how an individual researcher or journal acts. An individual researcher can have a very large h-index and have never given a single thought to their citation rate but there are also individual researchers for whom maximising that value is a core career goal. No judgement is made here either way, but there is significant external pressure from multiple directions (e.g. funding bodies and institutional policies) on both individuals and journals to maximise these metrics that impact on publication behaviour by individuals and, more importantly for this article, policy decisions by journals themselves.

All of these numerical metrics are proxies to the true value of a particular journal, a particular research paper or a particular researcher. Much to the probable chagrin of those who seek to quantify research capacity, the true value of a researcher's work or a scientific journal is not something that is easily boilied down to a small set of numerical indices. There is likely also a strong social communal element not captured by publication statistics. The true value of a publication is likely to be unknown for quite some time (perhaps decades beyond the passing of the researcher).

For those who have been working in the field for a while, it would not be hard to find examples of researchers who have equivalent h-indices and who publish in equivalently impactful journals, but whose work clearly differs in importance, impact and magnitude. It is also the case that peer review itself, particularly in grants, beyond a certain threshold value of any of these metrics, can lose discriminatory meaning. For instance, a reviewer might have some concern over an early-career researcher with a h-index of 5 who might be applying for a relatively large sophisticated grant, but would struggle to make a distinction between competing applicants with a h-index of 15 and 40 solely on the metric alone.

A typical astute researcher in the field may not necessarily trust a journal because it has a high impact factor or a researcher because they have a high h-index. The researcher would certainly take time to flip through the articles in the journal, getting a feel for the topics, examining whether there are fellow trusted colleagues and acquaintances (or competitors) publishing there and make a gut-level decision as to whether it is a predatory or for-profit journal (e.g. Beall 2015). It is unlikely that a researcher would make a decision on a journal's impact factor alone (despite potentially being externally pressured to).

Moreover, having such a focus on accepting only "highly citable" articles prevents the publication of useful articles in high-end journals that are not necessarily meant to be "highly citable" but are still useful. This includes, but is not limited to, such things as null results, "observing lore" which can provide practical methodology, replication studies, historical articles, project outlines, observatory classifications and simple deep case studies. Providing a places for articles such as these, and others, is part of the motivation for the creation of this journal.

\section{Journals that already exist}

\section{Subdiscipline-specific journals}

There are already avenues for publication in disciplinespecific journals. The most notable being the journal of the American Association of Variable Star Observers (JAAVSO, Percy 2017, weblink) which is a peer-reviewed publication open to submissions on various topics surrounding variable star research and observations, both archival and original, as well as educational and historical articles relevant to the field. There has been a long history of undergraduate and high school students publishing in the journal over the last few decades (e.g. Percy 2018) as well as a very active amateur community. For those not wanting to produce a full journal article, the 
AAVSO also runs a system allowing the upload of observations and measurements (weblink).

Other journals include the Journal of Double Star Observations (JDSO, Clark 2010; Freed et al. 2017, weblink) which captures the results and research endeavours of the pro-am double star community. The Minor Planet Bulletin (weblink) captures short peer-reviewed papers involving pro-am research on asteroids with a particular emphasis on asteroid rotational lightcurves. Two other journals that publish variable star observations and results are the Open European Journal on Variable Stars (OEJV: weblink) and Peremennye Zvezdy (weblink)

The Informational Bulletin on Variable Stars (IBVS, weblink) was another excellent place for variable star observation papers. Unfortunately, it closed down in 2019 not long after celebrating it's fiftieth year jubilee (Szeidl et al. 2011) citing human and IT resource requirements as being too large to rationalise the necessary resuscitation of the journal.

It can be seen from this list, that most journals at this level, by which is meant that they are not aimed at being the top tier of astronomical research, are focussed on a particular class of objects rather than astronomy in general. It is not known to the author whether a peer reviewed journal of similar nature and impact that has a broad scope on any particular class of objects or any generic topic in the field of astronomy. It is not a goal of ATOM to necessarily compete with already existing, wellestablished, discipline-specific journals.

\section{Non Subdiscipline-specific journals}

There are a number of publication opportunities with a variety of pro-am groups. There is the yearly conference proceedings of the Society for Astronomical Sciences (SAS, weblink) which provides opportunities to publish but isn't a journal in the traditional sense. Other examples are journals tied to astronomical societies, such as the Journal of the British Astronomical Association (JBAA, weblink) or the Journal of the Royal Astronomical Society of Canada (JRASC, weblink), which can occasionally feature research articles, although it is not their prime focus.

Historically, the Research Based Science Education (RBSE) project, published a student/teacher journal called the RBSE Journal, edited by Dr. Katy Garmany, from 1999 to 2010 (Hurst et al. 2008; Buxner 2014). This included the research of students and teachers involved in a variety of projects, including RBSE, ARBSE, TLRBSE, the Kitt Peak Teacher Observation Program and the SPITZER teacher observer program (Spuck et al. 2010), a precursor of the current NITARP (Rebull et al. 2018) program.

There is also the relatively new "Research Notes of the AAS" (RNAAS), which is also an attempt at a solution to the problem of a lack of simple, observational, minimal or null results in the astronomical literature (Vishniac and Lintott 2017). The AAS also publishes the peak Q1 Scopus journals, the Astronomical Journal and the Astrophysical Journal. However, RNAAS is not peer reviewed, accepts only short papers ( $<1000$ words), is not copyedited and only moderated by the editors rather than undergoing a detailed review process. This is intentional, particularly as the submissions are intended to be rapidly available online within 72 hours of receipt of the manuscript.

RNAAS is an exceptionally useful tool for the professional community in that it allows quick publication of results and ideas that may never have made it to print otherwise. However, it is perhaps not as useful for a beginning researcher who may want to publish a fully peer reviewed publication that is substantial in scope and length and whose scientific writing experience requires some scaffolding and support.

There are a variety of non-astronomy journals that accept specifically student work at the high school or undergraduate level (eg. The Journal of Undergraduate Research and The National High School Journal of Science), however it is very unlikely that research published in these journals will easily be discovered by other astronomers given their non-discipline specific nature. It has also been reported that some of these more generic education outlet focussed journals (as opposed to actual research journals) have rejected astronomy articles 
because they "don't fit the scientific method" (e.g. Tock 2019), even when the article is about a refined method of pulsar detection.

\section{What is the scope of ATOM?}

These publication metrics and the current range of similar journals show that there is a gap needed to be filled by a generic astronomy research journal. The journal is not aimed at publishing cutting edge research but research of use and of interest not necessarily of moderate to high impact. Anything that is a valid, new and useful contribution to the science of astronomy and related fields is acceptable, however small. This is not limited to but includes the following:

- Preliminary or speculative research (especially where the researcher may not continue to pursue the object of interest).

- Unconfirmed but potential discoveries

- Null results (including warnings of probable null results where research was cancelled due to this.)

- Observing lore that has not been published but has usually been 'handed down'

- Replication studies of previous research (within reason)

- Useful contributions from non-optimal instrumentation

- Heavily data-based contributions (as long as there is a good rationale for it being heavily data, rather than interpretive)

- Review articles, small and large, of patches of the sky, patches of the universe, interesting subsets of astronomical objects or discoveries or patches of the scientific literature.

- Historical articles

- Case studies
- Instrumentation design and calibration studies.

- Software design

- Tutorials for observing techniques and data analysis

- Detailed information about new projects, observatories and sites

- Computational astronomy and visualisation

- Theoretical modelling

- Outlines of methodology

The journal is open access. There are no page charges. There are no page limits, figure limits or reference limits. This does not mean "unlimited", it still needs to be concise, dense and to the point and provide enough information to pass peer review.

The first suspicion that someone may have upon encountering the journal for the first time might be to consider the possibility that it is predatory journal or something nefarious along those lines. This is not the case. If this was a predatory journal, it is a poor predator. There are no page charges, there are no publication charges, all work is voluntary. If the journal was nefarious or criminal at all, then it would be a poorly constructed endeavour of this type. This is a community endeavour to fill a need.

\section{The contrast between ATOM and a mainstream astronomy journal}

To illustrate the contrast, A\&A (Bertout and Schneider 2004) use an example of where they refused a paper... " that presented standard photometry for an unremarkable binary eclipsing star together with a standard interpretation of its light curve.". A\&A claim that they did not publish it because there was "insufficient content and scientific interest by today's standards" despite "both observations and interpretation were sound" to justify publication in A\&A. As ATOM is not aiming for impact at the cutting edge, this type of paper would be acceptable. The approach A\&A takes is typical of most 
mainstream top quartile journals and makes much sense for that context.

Sometimes research instrumentation of sufficient quality is not accessible to authors with less resources or less background to make measurements as precise as the best in the field. For instance, some measurements may be taken much closer to sea level than might be preferred. As long as the best has been made out of the best instrumentation available and the outcoming results have been made with scientific rigour with no major problems, it is acceptable for publication in ATOM.

The journal does not discriminate between single object studies or multi-object studies. For instance, Kepler found thousands of eclipsing binaries in it's FoV. Following up a single binary from that catalogue is perfectly acceptable. It is preferred, but not required, that this will be undertaken in great detail. Taking the Kepler database of eclipsing binaries and exploring it in a new direction is also acceptable. As is following up 15 of the binaries. What is preferred though is that if only one object is examined, it is done to far greater detail, includes a larger exploration of how the object fits into our general understanding or provides much greater novelty than a multi-object study.

\section{What are the requirements for a paper?}

The author requirements can obviously change over time but this particular paper remains static so it is best to check the "Instructions for Authors" at the site. Initially, however, the following requirements will be held:

- The journal requires authors to significantly connect with the literature. Science is not done in a vacuum but is a building upon of previous work and a networking of current work. The primary way that knowledge is linked at this stage in human history is by connecting relevant articles via referencing.

- Original data can be provided so that research can be picked up, re-analysed and forwarded, particularly as it is intended that preliminary studies are valid to accepted.

- The article *must* be written in LaTeX. This is the standard format of a scientific article. It can be quite daunting at first to a new LaTeX user, but online tools have made this a much simpler feat nowadays. An online tutorial will be available on the site for first time LaTeX authors.

- The submission has not been previously published, nor is it before another journal for consideration.

\section{The peer review process}

The Chief Editor is always the single person responsible for the publications within the journal. They are helped with the process via peer review as well as an editorial board. Not all publications submitted will automatically be sent to review. The Editor makes a first judgement of whether the research or the writing is of sufficient quality and acceptable topic or nature to go out to review. Ample consideration will be made for writers of English as a second language.

As typical articles may be from early career, student or novice authors, a developmental review may be undertaken before a full peer review if the editor so chooses. This is primarily to give guidance on how to work up the paper into a state where it is appropriately ready for a full peer review. It is likely that this developmental review will be very common as it is a stated goal of the journal to accomodate authors who are not career scientists and for whom writing a paper is a very new and unfamiliar process and whom are not already fluent in scientific writing.

Peer review is a very necessary tool in quality control. No article will be hastened through peer review and no article accepted without adequate and careful response to reviewers. The intended timeframe, while noting that firm deadlines are not possible in academic publishing, would be:

- One week for an initial editor appraisal 
- If required, a developmental review will take place over two weeks of frequent feedback and response.

- Once the article is ready for formal review, the intended timeframe to receive first reviews back is 1 month

- The timeframe from this point on is dependant on adequate responses from the author and whether further review rounds are necessary

Referees will be selected from active astronomy researchers who have published in the field on the topic in the past three years. In the tradition of most astronomy research journals, this is initially one blind reviewer. Extra reviewers may be brought in if there is a disagreement between the author and that reviewer or if the paper covers multiple fields.

\section{Considerations for a potential author}

Due to the wider scope of the journal and the permissiveness of preliminary and null results, this will likely lead to a lower "impact factor" for the journal. This very raw quantitative measure is used to compare the "performance" of journals within a particular field. It is, of course, very difficult to estimate how often a particular paper may be cited ahead of time. However, it can reasonably be assumed that a journal that is inclusive of more broader, speculative or null-reporting research is invariably going to publish a much higher number of articles with low citation rates. It is explicitly not a goal of this journal to achieve a high impact factor. Setting such a goal would be contrary to the mission of the journal to include valid papers with potentially low citation rates.

Hence this means that all quantitative metrics that measure a researcher's scientific output as a function of journal impact factor will score articles in this journal relatively low (we suspect). On the brighter side is the fact that most current metrics have moved beyond the inclusion of a journal's impact factor as a metric for publications of individual researchers (e.g. San Francisco Declaration on Research Assessment, Cagan 2013, link to DORA website). The h-index (Hirsch 2005), the current main quantitative rough estimate of a researcher's publication worth, does not care about impact factor at all, just numbers of publications and numbers of citations of those publications within the considered database. Many major funding bodies (Forschungsgemeinschaft 2010, press release) having seen people 'game' the system are moving away from using quantitative-based research appraisals entirely. Having said that, it is up to each author to find out what their institution or current or future grant funding body might value and act accordingly.

There can be no guarantee that this journal will be listed in Scopus or Web of Science curated indices. These two indices are the primary tools major institutions use to estimate publication impact. Initially, these indices do not consider "new" publications until they have a few years of track record and a calculable impact factor (which by definition requires at least two years of journal issues). We are aiming to meet the requirements of both Scopus and Web of Science but there is no guarantee of eventual selection, although we are aiming to comply with the requirements. Articles will be indexed via CrossRef and via Google Scholar from day one.

Having said all this, for many potential authors, particularly student researchers and pro-amateurs, considerations about such things as whether it counts for promotion or grant funding are largely irrelevant. For undergraduates, high school students and pro-ams, there is minimal functional difference between the Astronomical Journal, Proceedings of the Society for Astronomical Sciences, Proceedings of the Astronomical Society of the Pacific, RNAAS, JAAVSO and ATOM. What is of most benefit to them is the developmental peer review and the capacity to formally publish their valid scientific results in a scientific journal. In contrast, the extrinsic reward value of a publication is not as significant as the intrinsic personal development value, e.g. broadening perspectives and personal transformation (Beltzer-Sweeney and White 2019), content knowledge and process understanding, de- 
velopmental of scientific identity and community of practice membership (e.g. Freed 2019), of undertaking the scientific process in it's entirety from initial idea to final publication.

It is also the case that there is a significant time limitation on such authors. For a student or pro-am, typically the research undertaken is a single piece of research at a single epoch of time. In contrast, a professional or graduate student will likely be playing a longer game with perhaps tens of research streams that may formulate (or not) into research papers regularly over time on an indeterminate timescale. As the student or pro-am may be undertaking a research project once in a single restricted timeframe, they do not have the luxury of just letting a project take a backseat while they work on other projects. It is typically the one singular project they are undertaking and perhaps in a limited timeframe, e.g. a year or a semester for a student or when their observatory is not in the cloudy part of the year for a pro-am. These issues make the publication decisions and considerations for these potential authors markedly different than for a professional author. In turn, this influences the policies for journals, like those for ATOM outlined above, that would like to welcome such authors to publish.

\section{Conclusion}

This paper outlined the rationale for a new peer reviewed journal that provides a place for minimal to moderate impact papers in astronomy to be published. The journal focusses on the development of the author and the field in the process. It also provides a place for non-traditional outputs, such as observing lore, historical articles and observatory classifications. While there are good journals already that deal with object, or class of object, specific fields of research, this journal aims to capture, with peer review, any generic topic in astronomy.

\section{References}

Backhaus, U., Bauer, S., Beuermann, K., Diese, J., Dreizler, S., Hessman, F., Husser, T.-O., Klapdohr, K.-H., Möllmanns, J., Schünecke, R., et al. (2012). The quest for companions to postcommon envelope binaries-I. Searching a sample of stars from the CSS and SDSS. Astronomy \& Astrophysics, 538:A84.

Beall, J. (2015). Behind the Spam: A "Spectral Analysis" of Predatory Publishers. Proceedings of the International Astronomical Union, 11(A29A):166-171.

Beltzer-Sweeney, A. and White, S. L. (2019). The Double STARS Research Seminar: An analysis of its effects and methodologies. RTSRE Proceedings, 2(1).

Bertout, C. and Schneider, P. (2004). Editorship and peer-review at A\&A.

Beuermann, K., Buhlmann, J., Diese, J., Dreizler, S., Hessman, F., Husser, T.-O., Miller, G., Nickol, N., Pons, R., Ruhr, D., et al. (2011). The giant planet orbiting the cataclysmic binary DP Leonis. Astronomy \& Astrophysics, 526:A53.

Beuermann, K., Diese, J., Paik, S., Ploch, A., Zachmann, J., Schwope, A., and Hessman, F. (2009). A long-term optical and X-ray ephemeris of the polar EK Ursae Majoris. Astronomy \& Astrophysics, 507(1):385-388.

Buchheim, R. (2007). The sky is your laboratory: advanced astronomy projects for amateurs. Springer Science \& Business Media.

Buxner, S. R. (2014). Exploring how research experiences for teachers changes their understandings of the nature of science and scientific inquiry. Journal of Astronomy \& Earth Sciences Education (JAESE), 1(1):53-68.

Cagan, R. (2013). The San Francisco Declaration on Research Assessment. Disease Models \& Mechanisms, 6(4):869-870.

Clark, R. K. (2010). All about the journal of double star observations. Small Telescopes \& Astronomical Research. Collins Education Foundation, Santa Margarita, California. 
Conti, D. M. (2018). A Practical Guide to Exoplanet Observing.

Cutts, K. R. (2018). Astronomy student research in the international baccalaureate. RTSRE Proceedings, 1(1).

Fitzgerald, M. T., Criss, J., Lukaszewicz, T., Frew, D. J., Catelan, M., Woodward, S., Danaia, L., and McKinnon, D. H. (2012). RR Lyrae stars in the globular cluster NGC 6101. Publications of the Astronomical Society of Australia, 29(1):72-77.

Fitzgerald, M. T., Hollow, R., Rebull, L. M., Danaia, L., and McKinnon, D. H. (2014). A review of high school level astronomy student research projects over the last two decades. Publications of the Astronomical Society of Australia, 31.

Fitzgerald, M. T., Inwood, L., McKinnon, D. H., Dias, W., Sacchi, M., Scott, B., Zolinski, M., Danaia, L., and Edwards, R. (2015). Photometric and proper motion study of the neglected open cluster NGC 2215. The Astronomical Journal, 149(6):190.

Forschungsgemeinschaft, D. (2010). Quality not quantity. DFG adopts rules to counter the flood of publications in research. Press release.

Freed, R. (2018). Astronomy Research Seminar: The Impact on Students from their Perspective. Preliminary results from one spring seminar. $R T$ SRE Proceedings, 1(1).

Freed, R. (2019). Astronomy Research Seminar Expansion and Building a Community-of-Practice. RTSRE Proceedings, 2(1).

Freed, R., Fitzgerald, M., Genet, R., and Davidson, B. (2017). An overview of ten years of student research and JDSO publications. In Society for Astronomical Sciences Annual Symposium, volume 36, pages 131-136.

Frew, D. J., Stanger, J., Fitzgerald, M., Parker, Q., Danaia, L., McKinnon, D., Guerrero, M. A., Hedberg, J., Hollow, R., An, Y., et al. (2011). K 1-6: an asymmetric planetary nebula with a binary central star. Publications of the Astronomical Society of Australia, 28(1):83-94.

Gomez, E. L. and Fitzgerald, M. T. (2017). Robotic telescopes in education. Astronomical Review, 13(1):28-68.

Guieu, S., Rebull, L., Stauffer, J., Vrba, F., NoriegaCrespo, A., Spuck, T., Moody, T. R., Sepulveda, B., Weehler, C., Maranto, A., et al. (2010). Spitzer observations of IC 2118. The Astrophysical Journal, 720(1):46.

Hirsch, J. E. (2005). An index to quantify an individual's scientific research output. Proceedings of the National academy of Sciences, 102(46):16569-16572.

Howell, S. B., Brinkworth, C., Hoard, D., Wachter, S., Harrison, T., Chun, H., Thomas, B., Stefaniak, L., Ciardi, D. R., Szkody, P., et al. (2006). First Spitzer Space Telescope observations of magnetic cataclysmic variables: Evidence of excess emission at 3-8 $\mu \mathrm{m}$. The Astrophysical Journal Letters, 646(1):L65.

Howell, S. B., Hoard, D., Brinkworth, C., Kafka, S., Walentosky, M., Walter, F. M., and Rector, T. (2008). "Dark Matter" in Accretion Disks. The Astrophysical Journal, 685(1):418.

Hurst, A., Garmany, K., Steiner, R. V., Connolly, R., Buxner, S., and Gomez, E. (2008). Providing Professional Development from a Distance: The Opportunities and Challenges of Reaching Educators through Online Astronomy Courses. In EPO and a Changing World: Creating Linkages and Expanding Partnerships, volume 389, page 23.

Percy, J. (2017). JAAVSO: Past, Present, and Future. JAVSO, 45(2):131.

Percy, J. (2018). Forty Years of Linking Variable Star Research with Education. RTSRE, 1(1):95104.

Rebull, L., Fitzgerald, M., Roberts, T., French, D. A., Laurence, W., Gorjian, V., and Squires, 
G. K. (2018). The NASA/IPAC Teacher Archive Research Program (NITARP). RTSRE, 1(1):171187.

Rebull, L., Johnson, C., Hoette, V., Kim, J., Laine, S., Foster, M., Laher, R., Legassie, M., Mallory, C., McCarron, K., et al. (2011). New young star candidates in CG4 and Sa101. The Astronomical Journal, 142(1):25.

Spuck, T., Pompea, S., Rebull, L., Gorjian, V., Howell, S., Johnson, C., Kennedy, S., and Thomas, B. (2010). Impact Summary: The Spitzer Space Telescope Research Program for Teachers and Students. ApJ, 685:418-427.

Swift, J. and Vyhnal, C. (2018). The Astronomy Program at the Thacher School. RTSRE, 1(1):281-282.

Szeidl, B., Sterken, C., Oláh, K., Jurcsik, J., Holl, A., and Szabados, L. (2011). Jubilee Issue of IBVS: Half a Century of Variable Star Science Publishing. Information Bulletin on Variable Stars.

Tock, K. (2019). Astronomy, Technology, and the Scientific Method:Reflections on the Astronomy Research Seminar at Stanford Online High School, Spring 2018. RTSRE Proceedings, 2(1).

Vishniac, E. T. and Lintott, C. (2017). The Research Notes of the American Astronomical Society. Research Notes of the AAS, 1(1):1. 